\title{
Physiotaping beim Pferd
}

\section{So können Patellabeschwerden und Spat gelindert werden}

Kerstin Rudde und Sören Heinbokel

\section{จ Zusammenfassung}

Im Rahmen einer ganzheitlichen Betreuung von Pferd und Reiter wurden erstmals sogenannte Physiotape-Anlagen für das Sportpferd entwickelt. Anhand zweier detaillierter Beispiele zur Therapie von Spat bzw. Patellabeschwerden wird der erfolgreiche Einsatz des Physiotapes aufgezeigt und die spezifische Anlagetechnik dieser schonenden Therapiemethode erläutert.

Ob Hochleistungs- oder Freizeitsportler: Bei Muskel- und Sehnenbeschwerden werden beim Menschen bereits seit vielen Jahren erfolgreich sogenannte Physiotapes nach kinesiologischen Grundlagen angewandt. Das Einsatzgebiet ist dabei vielseitig: vom Tennisarm (Epicondylitis radialis humeri) über Achillessehnenüberlastung (Achillodynie) bis hin zu Rückenschmerzen, Schulter- oder Knieinstabilitäten die Tapes führen zu einer Schmerzlinderung und Steigerung der Belastbarkeit.

\section{Unterschied zu herkömmlichen Tapes}

Im Unterschied zu herkömmlichen Tapes ist das Material der Physiotapes dehnbar und elastisch, wodurch die volle Beweglichkeit ohne Bewegungseinbußen erhalten bleibt. Das Material wurde dabei der Haut nachempfunden. Ein zusätzliches Plus des Materials sind die lange Tragedauer (4-7 Tage) und die Wasserbeständigkeit bei korrekter Anlage. Dies konnten Versuche des deutschen Schwimmverbandes bestätigen [1].

\section{Welchen Effekt erzielen Physiotapes?}

\section{Aktivierung der Zirkulation}

Nach einem Trauma entsteht durch Austreten von Lymphe in das umgebende Gewebe eine Druckerhöhung. Die Schwellung behindert zudem den Lymphabfluss. Beim Anlegen der Physiotapes werden Hautfalten (Convolutions) gebildet, die zu einer Druckminderung und schließlich zu einem Abfluss der Lymphflüssigkeit führen.

\section{Verbesserung der Muskelfunktion}

Durch das Anlegen von Physiotapes wird den Muskeln eine Zugrichtung vorgegeben. Mechanorezeptoren werden gereizt, was zu einer Änderung der Muskelspannung führt.

\section{Unterstützung der Gelenkfunktion}

Die Gelenkfunktion wird auf mehreren Ebenen unterstützt. Zum einen verschiebt jede Bewegung des Körpers über die Convolutions die Haut und führt somit zu einer Stimulation der Mechanosensoren der Haut. Gleichzeitig verleiht das Physiotape dem Gelenk eine passive Stabilität.

\section{Physiotape-Anlagen beim Pferd}

Um eine optimale Wirkung zu erzielen, ist vor der Anlage eine intensive Befundung notwendig, um die richtige Anlagetechnik für das Beschwerdebild festzulegen. Es wird zwischen Muskel-, Ligament-, Faszien-, Korrektur- und Lymphtechniken unterschieden. Die verschiedenen Wirkweisen werden über Veränderungen der Dehnung und Zugrichtung erzielt.

Im Rahmen einer ganzheitlichen Betreuung von Pferd und Reiter haben die Autoren erste Anlagen für das Sportpferd entwickelt. Diese zeigen durchweg posi- tive Effekte und sind problemlos in ein Therapiekonzept sowohl bei Sport- als auch bei Freizeitpferden integrierbar.

Es wird ausdrücklich der Besuch einer speziellen Physiotaping-Fortbildung und die Zusammenarbeit mit einem Veterinär, einem Physiotaping-Experten und/oder geschulten Physiotherapeuten empfohlen.

\section{Vorbereitung des Patienten}

Da Pferde im Gegensatz zum Menschen Fell besitzen, wird empfohlen, die Haare über den zu beklebenden Strukturen schmutz- und fettfrei zu halten. Am einfachsten gelingt dies durch Waschen mit einem Pferdeshampoo. Anschließend sollte das Fell getrocknet und mit Kardätsche und Striegel gründlich gebürstet und langes Fell ggf. geschoren werden.

Der Einsatz von PINOFIT PHYSIOTAPE wurde tierärztlich empfohlen, da es hervorragende Klebeeigenschaften am Tier besitzt [2].

\section{Anlage eines Physiotapes bei Spat}

Spat ist eine arthritische Entzündung am Sprunggelenk des Pferdes. Ziel der Tapeanlage bei dieser Erkrankung ist vor allem eine Entlastung der Sehne (Tendo calcaneus). Zusätzlich wird die umgebene Muskulatur detonisiert. Durch das „Anheben“ der Haut (Bildung von Convolutions) werden zudem die natürlichen Heilungsprozesse unterstützt.

\section{Technik}

Für die Anlage wird das betroffene Bein je nach Größe des Pferdes in die Beugung genommen oder der Huf auf eine Erhöhung gesetzt. Es wird ein $5 \mathrm{~cm}$ breiter, nicht eingeschnittener Tapestreifen (bezeichnet als I-Zügel) am Tuber calcanei des Calcaneus angesetzt und mit maximalem Zug 

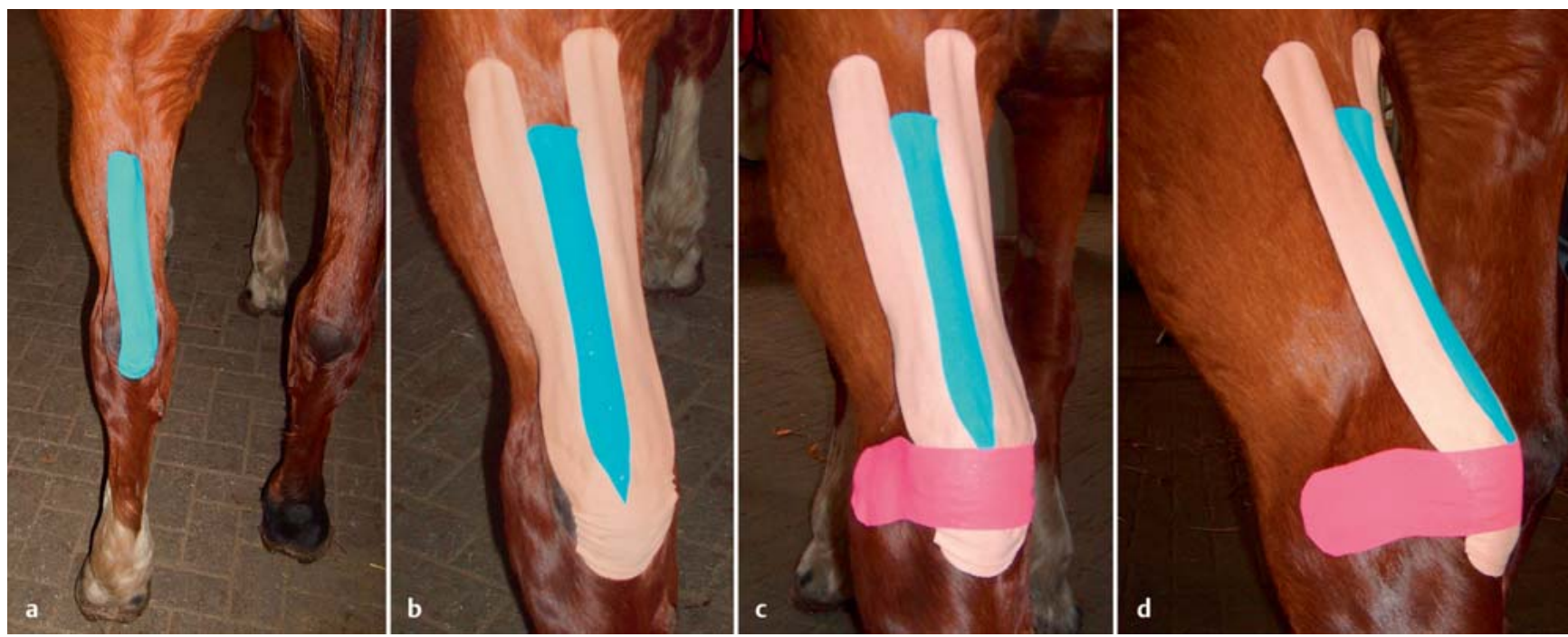

Abb. 1 a bis d Anlage eines Physiotapes bei Spat. Die Autoren empfehlen die Anlage mit zwei Therapeuten, wobei einer den Zügel verklebt, die zweite Person den Lauf beugt. a Anlage des I-Zügels (blau) ausgehend vom Calcaneus. Er wird mit maximalem Zug getapet. b Anlage des Y-Zügels (beige) über den Tractus calcaneus lat. und Tractus calcaneus med. Sichtbar ist außerdem der vorher angelegte I-Zügel (blau). c und d Aufsicht auf die fertige Physiotape-Anlage von kaudal (c) bzw. lateral (d). Neben den I- und Y-Zügeln ist nun auch die abschließende Fixation mithilfe eines weiteren I-Zügels (pink) sichtbar.

über den Verlauf der Sehne nach oben geklebt (\$ Abb. 1 a). Die Länge wird individuell angepasst und ist abhängig von Größe und Schmerzlokalisation. Generell laufen alle Zügel auf den letzten Zentimetern ohne Zug aus.

Als nächster Schritt wird ein Tapestreifen als Y-Zügel parallel zum vorab fixierten Zügel gelegt (\$Abb.1 b). Bei diesem Zügel ist die eine Seite des Tapestreifens wie bei einem Yeingeschnitten. Als Orientierung kann hierbei die Bursa subcutanea calcanea dienen, von der der Tapestreifen über den Tractus calcaneus lat. und Tractus calcaneus med. mit leichtem Zug nach oben geklebt wird.

Das letzte Tape dieser Anlage wird als I-Zügel mit maximalem Zug zur Stabilisierung kaudal um das Sprunggelenk gelegt (\$Abb. $1 \mathrm{c}$ und d).

Auf eine ausreichende Beugung des zu behandelnden Beins ist zu achten. Alternativ kann das Bein auf eine Erhöhung gestellt werden, sofern das Tier dies zulässt.

\section{Anlage eines Physiotapes bei Patellabeschwerden}

Eine weitere effektive Anlage ist im Bereich der Patella äußerst erfolgreich anwendbar. Die Patella bildet mit dem Kniescheibenknorpel und mit ihren Kniescheibenbändern (Lig. patellae) die Patellaschlaufe. An der belasteten Gliedmaße gleitet die Patel-

\section{Tipps zur Anlagetechnik}

- Es ist darauf zu achten, dass bei allen Tapestreifen die Ecken abgerundet werden, um ein frühzeitiges Ablösen zu verhindern.

- Durch das Erzeugen von Reibungshitze durch schnelle Streichbewegungen über die Tape-Zügel wird die temperatursensitive Schicht des Klebers aktiviert, sodass er seine Wirkung entfalten kann. Nach einer Stunde sitzt die Anlage sicher am Pferd und widersteht auch starken Belastungen, Schweiß

la labil auf dem medialen Rollkamm der Trochlea ossis femoris (Tuberculum trochlea ossis femoris). Allein durch den Muskeltonus des M. quadriceps femoris wird ein Abgleiten vom Rollkamm verhindert und die Winkelhaltung im Kniegelenk des Standbeins gehalten. Ziel des Tapings ist es, die Patella zu stabilisieren.

\section{Technik}

Die Anlage ist verhältnismäßig einfach. So wird ein Y-Zügel von proximal nach distal im Verlauf lateral um die Patella mit maximalem Zug gelegt ( Abb. 2a). Trotz eventuell vorhandener Fellwirbel ist eine Anlage problemlos möglich, sofern der Kleber im Tape durch Reibungshitze aktiviert wird. Teilweise entsteht der Eindruck, dass das Tape nicht hinreichend verklebt ist. Dieser optische Eindruck täuscht, da und Regen. Bis dahin ist das Pferd zu schonen bzw. in Boxenruhe zu halten.

- Die Anlagen verbleiben so lange auf dem Fell, bis diese beginnen sich abzulösen. Trotz der starken Klebekraft sind die Streifen jederzeit mit sanftem Zug problemlos und ohne Schmerzen für das Tier wieder zu entfernen.

- Sollten sich die Streifen schlecht entfernen lassen, wird empfohlen, die Anlage mit Seifenlauge zu waschen.

durch die gewollte Bildung der Convolutions die Anlage etwas wellig erscheint.

Der M. quadriceps femoris (M. vastus femoris) wird mit in die Klebung eingeschlossen. Zwei I-Zügel medial und lateral umschließen in Ligamenttechnik die Patella ( Abb. 2 b und c). Bei der Anlage sollte das betroffene Bein leicht erhöht gestellt werden, um den Muskel in entsprechende Vordehnung zu bringen.

Wir haben die Erfahrung gemacht, dass die Anwendung beispielsweise im Bereich der Kruppenmuskulatur als detonisierendes Physiotaping des M. semimembranosus durch I-Zügel möglich ist.

Ebenfalls positive Erfahrungen bestehen in der Therapie des M. trapezius und der dorsalen, autochthonen Wirbelsäulenmuskulatur, speziell der Mm. longissimus und multifidii. 

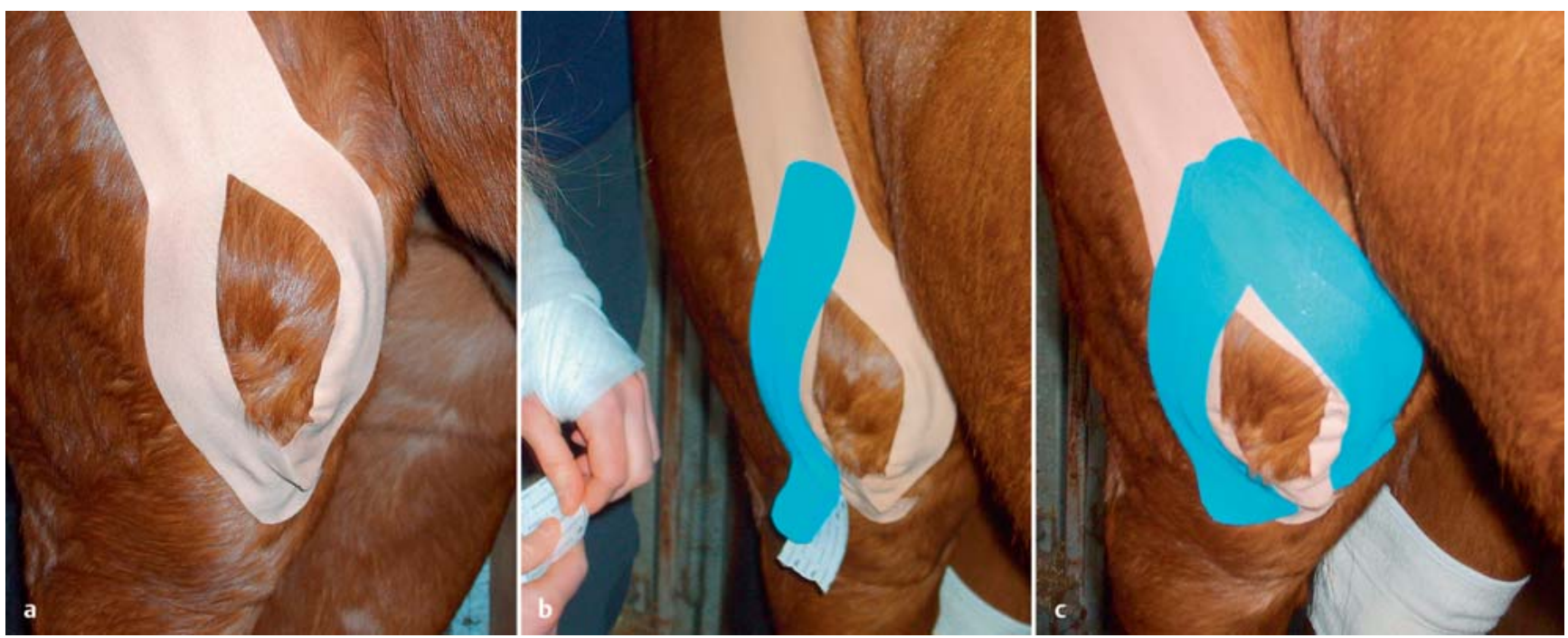

Abb. 2a bis c Anlage eines Physiotapes an der Patella. a Anlage eines breiten Y-Zügels (beige) um die Patella. Die Anlage erfolgt in leichter Beugung des Beins. b Die Anlage der blauen I-Zügel kann ohne Beugung des Beins erfolgen, diese werden stabilisierend zum Teil über den Y-Zügel angebracht. c Eine fertige Anlage

\section{Blick in die Zukunft des Physiotapings}

Weitere Tapeanlagen werden derzeit unter unserer Leitung in einem interdisziplinären Team aus Physiotherapeuten und Veterinären erprobt.

Die Untersuchung des Muskeltonus vor, während und nach der Tapeanlage durch Myogrammableitung mit zusätzlichen statistischen Tests und die Untersuchung der osteopathischen Einflüsse sollen weitere wichtige Rückschlüsse für die erfolgreiche Anlage von Physiotapes im Pferdesport ermöglichen.

\section{Danksagung}

Wir möchten uns besonders bedanken bei PT A. Fink für die Unterstützung bei den Tapeanlagen und bei der Erstellung der Fotos. Zudem gilt unser Dank THP DIPO A.M. Weingart, PT L. Gutkin und C. Boss für die vielen guten Ideen und den fachlichen Rat bei den Tapeanlagen. $\bullet$

\section{๑) Summary}

\section{Physiotaping in horses - a way to ease pain caused by patella problems and bone spavin}

Physiotaping in horses is a new and promising technique, developed in the context of an integrated supervision of horse and equestrian. The successful implementation and specific application of this gentle therapeutic method, focussing in particular on patella problems and bone spavin, are described in detail in this article.

\section{๑) Key words}

horse - kinesiology - taping - PHYSIOTAPE

\section{(- Literatur}

[1] Habib K: Pinofit Physiotaping - Veränderung der Schmerzsymptomatik im Wasser bei Leistungsschwimmern. Bachelorarbeit, 2008, in Druck.

[2] Unkrig AS, Weingart AM: Die Anwendung verschiedener Tapematerialien am Sportpferd. Unveröffentlichte Rohdaten, 2007.

\section{Kerstin Rudde und Sören Heinbokel}

Sport \& Reha Zentrum - die Gesundheiter Duesbergstraße 1

46325 Borken

E-Mail: equestrian@heinbokel.de

www.heinbokel.de

www.sport-reha-zentrum.de
PT Kerstin Rudde, geb. in Borken (Westfalen), Ausbildung zur Physiotherapeutin in Münster. Seitdem vielfältige Aus- und Weiterbildungen z.B. Lymphdrainage und manuelle Therapie, Pilates und AMM, zertifizierte PINOFIT PHYSIOTAPEExpertin. Seit August 2001 angestellt im Sport \& Reha Zentrum - die Gesundheiter in Borken, hier u. a. Fachbeauftragte Sporttaping. Ganzheitliche Betreuung von Leistungssportlern aus dem Handball- und Pferdesport.

THP HD Sören Heinbokel, geb. in Cuxhaven, Biologiestudium an der RWTH Aachen, Ausbildung zum Tierheilpraktiker in Köln und Aachen, mit Schwerpunkt Pferd. Zertifizierter PINOFIT PHYSIOTAPE-Experte. Seit Januar 2000 regelmäßig tätig für das Sport \& Reha Zentrum - die Gesundheiter in Borken, zudem Therapieleiter MaiaMedical/Horse-Group. Ganzheitliche Betreuung von Pferd und Reiter. Veröffentlichungen in Fachzeitschriften, u. a. für die Retterspitz $\mathrm{GmbH}$, vielfältige Einsätze als zoologischer Fachberater. 